\title{
CULTURA DE SEGURANÇA DO PACIENTE: PERCEPÇÕES E ATITUDES DOS TRABALHADORES DE CENTRO CIRÚRGICO
}

\author{
Patient safety culture: perceptions and attitudes of surgical center workers
}

\author{
Cultura de seguridad del paciente: percepciones y \\ actitudes de los trabajadores del centro quirúrgico
}

\author{
José Francisco da Silva Júnior ${ }^{1} \mathbb{D}$, Pedro José de Jesus Júnior ${ }^{2}$ (D) Thialla Andrade Carvalho ${ }^{3}$ (D), \\ Maria Pontes Campos de Aguiar ${ }^{4}$ (D), Simonize Cunha Barreto de Mendonça ${ }^{5}$ (D), Daniela de Souza Lordelo ${ }^{6}$ (D)
}

RESUMO: Objetivo: Avaliar a cultura de segurança por meio das percepções e atitudes dos profissionais que atuam no centro cirúrgico de um hospital de ensino. Método: Trata-se de um estudo exploratório, descritivo e transversal, com abordagem quantitativa, desenvolvido com 110 profissionais que atuam no centro cirúrgico, utilizando o Safety Attitudes Questionnaire. Resultados: A média geral dos escores evidenciou uma cultura de segurança incipiente. Ao avaliar a cultura por domínios, satisfação do trabalho e percepção do estresse foram avaliados como positivos, e clima de trabalho em equipe, clima de segurança, percepção da gerência da unidade/hospital e condições de trabalho, como negativos. Conclusão: O nível de cultura de segurança encontrado está abaixo do preconizado na literatura. As ações gerenciais e as condições de trabalho foram consideradas os principais fatores que contribuíram para a fragilidade dessa cultura, entretanto os profissionais demonstraram-se satisfeitos com a unidade de trabalho.

Palavras-chave: Cultura. Segurança do paciente. Centros cirúrgicos. Pessoal de saúde. Pesquisa sobre serviços de saúde.

ABSTRACT: Objective: To evaluate safety culture based on the perceptions and attitudes of professionals who work in the surgical center of a teaching hospital. Method: This is an exploratory descriptive cross-sectional study, with a quantitative approach, developed with 110 professionals who work in the surgical center, using the Safety Attitudes Questionnaire. Results: The general mean scores showed an incipient safety culture. When evaluating the culture by domains, job satisfaction and stress recognition were assessed as positive, and teamwork climate, safety climate, perceptions of unit/hospital management, and working conditions, as negative. Conclusion: The level of safety culture found is below that recommended in the literature. Management actions and working conditions were considered the main factors that contributed to the fragility of this culture. However, the professionals were satisfied with their work unit. Keywords: Culture. Patient safety. Surgicenters. Health personnel. Health services research.

RESUMEN: Objetivo: evaluar la cultura de seguridad, a través de las percepciones y actitudes de los profesionales que trabajan en el centro quirúrgico de un hospital universitario. Método: Estudio exploratorio, descriptivo y transversal, con enfoque cuantitativo, desarrollado con 110 profesionales que trabajan en quirófano, aplicando el Cuestionario de Actitudes de Seguridad (Safety Attitudes Questionnaire). Resultados: El promedio de la puntuación general mostró una incipiente cultura de seguridad. Al evaluar la cultura por dominios, la "satisfacción laboral” y la "percepción del estrés" se evaluaron como positivas, y el "clima de trabajo en equipo", el "clima de seguridad", la "percepción de gestión de la unidad/hospital" y las "condiciones de trabajo" como negativas. Conclusión: El nivel de cultura de seguridad encontrado es inferior al recomendado en la literatura. Las acciones de gestión y las condiciones de trabajo se consideraron los principales factores que contribuyeron a la fragilidad de esta cultura. Sin embargo, los profesionales estaban satisfechos con la unidad de trabajo. Palabras clave: Cultura. Seguridad del paciente. Centros quirúrgicos. Personal de salud. Investigación sobre servicios de salud.

'Graduado em Enfermagem (Bacharelado) pela Universidade Federal de Sergipe (UFS) - Aracaju (SE), Brasil.

2Graduado em Enfermagem (Bacharelado) pela UFS. Residente em enfermagem obstétrica pela Universidade Federal da Bahia - Salvador (BA), Brasil.

${ }^{3}$ Mestre pelo Programa de Pós-Graduação em Enfermagem da UFS. Enfermeira do Instituto Federal de Sergipe - Aracaju (SE), Brasil.

"Professora doutora do Departamento de Enfermagem da UFS - Aracaju (SE), Brasil.

${ }^{5}$ Doutoranda pelo Programa de Pós-Graduação em Ciências da Saúde da UFS. Enfermeira do Serviço de Controle de Infecção Relacionada à Assistência à Saúde do Hospital Universitário de Sergipe -Aracaju (SE), Brasil.

'Mestre pelo Programa de Pós-Graduação em Enfermagem da UFS. Enfermeira da Central de Material de Esterilização do Hospital Universitário de Sergipe - Aracaju (SE), Brasil.

*Autora correspondente: danylordelo@hotmail.com

Recebido: 24/09/2019 - Aprovado: 29/04/2020

https://doi.org/10.5327/Z1414-4425202000030003 


\section{INTRODUÇÃO}

A segurança do paciente, desde o ano de 2004, tornou-se um tema mundialmente discutido, com o objetivo de melhorar a qualidade da assistência à saúde, uma vez que busca reduzir os episódios de incidentes ocasionados pelos cuidados de saúde que resultam em danos ao paciente ${ }^{1}$. Essa temática tornou-se primordial com a criação da Aliança Mundial para a Segurança do Paciente, pela Organização Mundial da Saúde (OMS), que instituiu um conjunto de medidas, por meio de campanhas, voltadas para as boas práticas assistenciais denominadas de Desafios Globais para a Segurança do Paciente, com os propósitos de reforçar as práticas de segurança e promover melhor comunicação e trabalho da equipe multidisciplinar².

No Brasil, somente em 2013, com a criação do Programa Nacional de Segurança do Paciente (PNSP) e a publicação da Resolução da Diretoria Colegiada (RDC) n ${ }^{\circ}$ 36, a cultura de segurança foi considerada um dos princípios da gestão de risco voltada para a qualidade e a segurança do paciente. Entre as metas discutidas nesse programa, destaca-se a segurança do paciente cirúrgico como prioridade nos serviços de saúde ${ }^{3}$.

Salienta-se que o centro cirúrgico (CC) é um local que oferece assistência complexa por meio de equipes multiprofissionais e da alta densidade tecnológica, porém com elevado risco de danos ao paciente ${ }^{4}$. A probabilidade de ocorrência de danos pode estar associada ao estresse dos profissionais, à comunicação ineficaz, à alta carga de trabalho e / ou ao duplo vínculo empregatício. Diante disso, para o alcance da segurança do paciente cirúrgico, torna-se imprescindível a participação da equipe multiprofissional e dos atores organizacionais em relação à cultura de segurança do paciente 5 .

Define-se cultura de segurança como um produto de valores, atitudes, competências e padrões de comportamentos individuais e coletivos que determinam o compromisso, o estilo e a proficiência da administração de uma organização segura. Com os propósitos de compreender e avaliar a cultura de segurança, existem vários instrumentos em diversos idiomas que podem ser utilizados como ferramentas ${ }^{6}$.

No Brasil, o Safety Culture Surveyand (CSS) e o Safety Attitudes Questionnaire (SAQ) são os instrumentos mais utilizados nas instituições hospitalares ${ }^{7}$. Para o desenvolvimento deste estudo, escolheu-se o SAQ em uma versão adaptada para língua portuguesa, por ser confiável e sensível para avaliar atitudes e percepções individuais relacionadas à segurança ${ }^{8}$.

Estudos nacionais realizados nas regiões Sul e Nordeste com equipes multiprofissionais sobre a cultura da segurança dos pacientes em diferentes setores hospitalares e na atenção básica da saúde concluíram que a cultura de segurança pode ser influenciada pelo cargo do ocupante, pela carga horária de trabalho, pela gestão hospitalar e pelo estresse. Salienta-se que tais estudos foram realizados em unidades de terapia intensiva e internação, sendo evidente a necessidade de que a cultura de segurança seja mais bem esclarecida em unidades de CC, uma vez que este é um setor de alto risco.

\section{OBJETIVO}

Avaliar a cultura de segurança do paciente, por meio das percepções e atitudes dos profissionais que atuam no CC de um hospital de ensino do nordeste brasileiro.

\section{MÉTODO}

Estudo exploratório, descritivo e transversal, com abordagem quantitativa, desenvolvido no CC de uma instituição hospitalar pública e de ensino de Aracaju, Sergipe, Brasil.

Fizeram parte do estudo 110 profissionais, contemplando as categorias: médicos-cirurgiões, anestesiologistas, residentes de medicina, enfermeiros, técnicos em enfermagem, auxiliares de enfermagem, auxiliares de saúde (maqueiros, secretário de clínica) e técnico em farmácia.

Foram incluídos na investigação os profissionais que atuavam direta ou indiretamente na assistência e trabalhavam no setor havia pelo menos um mês, com carga horária mínima de 20 horas semanais. Não foram incluídos no estudo os profissionais que por qualquer motivo estavam afastados no período da coleta dos dados, por licença, férias e folga. No período da coleta nenhum profissional estava afastado de suas atividades laborais.

Utilizou-se para coleta de dados o Questionário de Atitudes de Segurança, do SAQ — Short 2006 — , traduzido e validado para língua portuguesa do Brasil. O SAQ é um instrumento que mensura o clima de segurança compreendido pelos profissionais, sendo dividido em duas partes. A primeira parte é composta de variáveis de caracterização do sujeito (cargo, sexo, especialidade, tempo na especialidade);. A segunda parte possui 41 questões, contemplando os domínios: clima de trabalho em equipe, satisfação no trabalho, percepção da gestão do hospital e da unidade, condições de trabalho e reconhecimento do estresse. As respostas a cada uma dessas questões seguem uma escala de Likert de 5 pontos:

- A: discordo totalmente;

- B: discordo parcialmente;

- C: neutro;

- D: concordo parcialmente; 
- E: concordo totalmente;

- X: não se aplica 9 .

Nessa parte, foram acrescidas as variáveis: idade, escolaridade, tempo de trabalho no setor (em anos), turno de trabalho, vínculos empregatícios, assistência ao paciente (direta ou indireta).

A coleta dos dados foi realizada de maio a julho de 2016, por três coletores. Os profissionais que aceitaram participar do estudo assinaram o Termo de Consentimento Livre e Esclarecido e foram explicitados sobre os propósitos do estudo. O questionário foi entregue para cada participante em um envelope fechado, que, após o preenchimento, foi depositado em uma pasta deixada no local, a fim de manter o sigilo dos participantes. Todas as pastas foram recolhidas no fim de cada turno de trabalho.

Os dados foram organizados e analisados no programa Epi info ${ }^{\circledR}$, versão 7, com dupla digitação independente. As variáveis demográficas e laborais quantitativas foram descritas por medidas de tendência central e dispersão, e as variáveis qualitativas categóricas, por estatística descritiva simples.

Para a análise do $S A Q$, avaliaram-se as 41 questões por meio de um escore que variou entre 0 e 100 pontos, sendo 0 a pior percepção do clima de segurança e 100 a melhor. As questões 2, 11 e 41 possuem escore reverso. Ou seja, nesses casos, 0 representa a melhor percepção de clima de segurança e 100 a pior percepção. Cada questão recebeu a seguinte pontuação:

- discordo totalmente (A): 0 ponto;

- discordo parcialmente (B): 25 pontos;

- neutro (C): 50 pontos;

- concordo parcialmente (D): 75 pontos;
- concordo totalmente (E): 100 pontos;

- não se aplica $(X)$ : não recebeu pontuação.

Valores com escores $\geq 75$ foram considerados positivos.

O presente estudo respeitou a Resolução no 466/2012, sobre pesquisas envolvendo seres humanos, e foi aprovado pelo Comitê de Ética em Pesquisa da Universidade Federal de Sergipe (Certificado de Apresentação para Apreciação Ética 50133315.8.0000.5546).

\section{RESULTADOS}

Entre os 110 profissionais que participaram do estudo, a mediana de idade foi de 35 anos. Em relação à carga horária de trabalho, foi encontrada mediana de 36 horas semanais, e quanto ao tempo de serviço no setor, a mediana foi de 18,5 meses. A maioria dos participantes é do sexo feminino (61/55,5\%). As categorias profissionais com maior número de representantes foram os médicos-cirurgiões e anestesiologistas, residentes de medicina com 32 sujeitos $(29,1 \%)$, seguidos por auxiliares de enfermagem e técnicos em enfermagem (27 / 24,5\%), enfermeiros (nove / 8,2\%), auxiliares de saúde e técnicos em farmácia $(10 / 9,1 \%)$. Em relação à escolaridade, 86 (78,2\%) tinham ensino superior completo e 64 (58,2\%) especialização.

$\mathrm{Na}$ análise por domínios, verificou-se que o domínio com a maior média foi percepção do estresse $(77,5 \%)$, enquanto percepção da gerência do hospital obteve a menor média $(55,1 \%)$. A média geral obtida foi $69,1 \%$ (Figura 1).

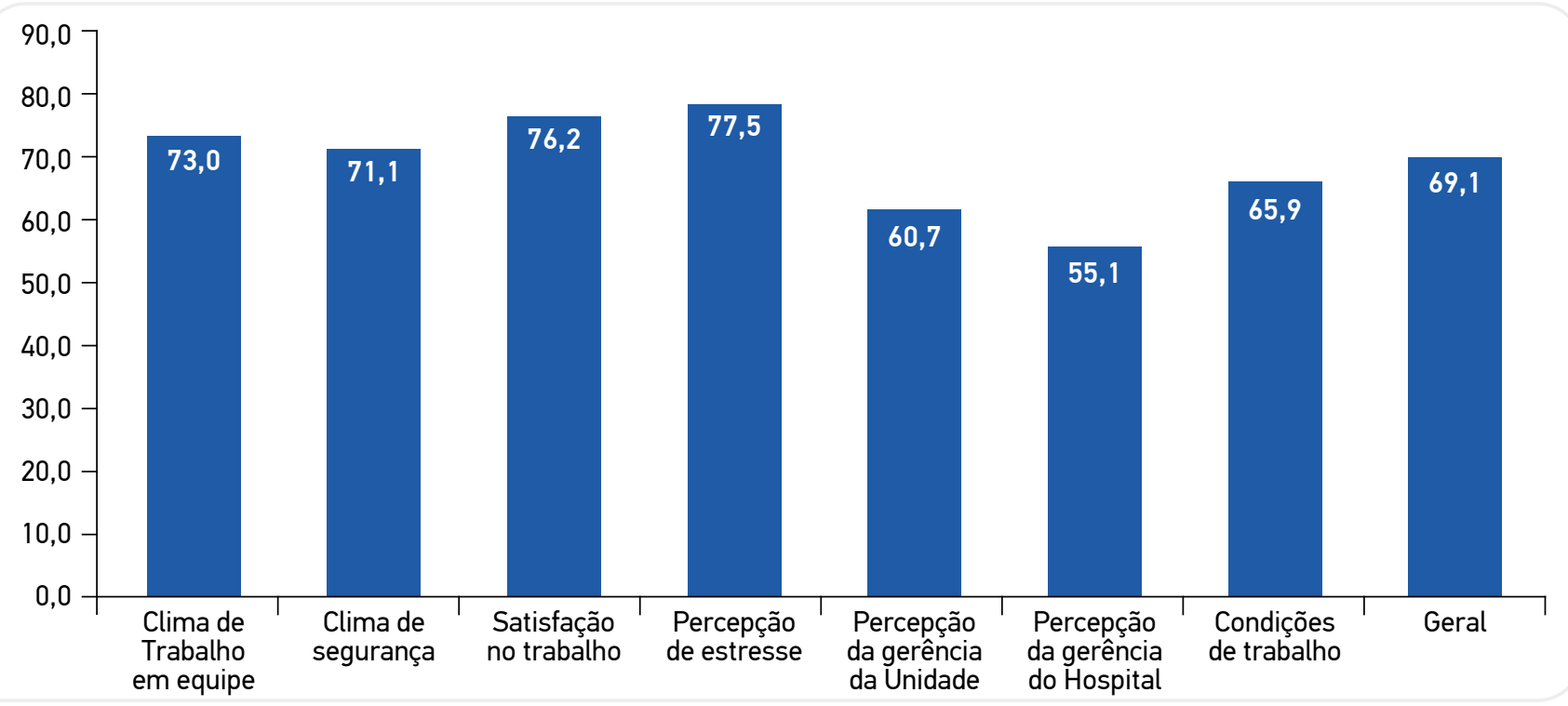

Figura 1. Média dos escores obtidos pela avaliação dos profissionais de saúde que trabalham no centro cirúrgico, segundo as dimensões do questionário Safety Attitudes Questionnaire. 
No domínio clima de trabalho em equipe, foi averiguado que 90 (82\%) profissionais concordaram com o item "Eu tenho o apoio de que necessito dos outros membros da equipe para cuidar dos pacientes", no entanto apenas 27 (25\%) concordaram com o item "Neste setor é difícil falar abertamente se eu percebo um problema com o cuidado ao paciente".

Em relação ao domínio clima de segurança, 23 (21\%) entrevistados discordaram da seguinte afirmativa: "Erros são tratados de maneira apropriada neste setor"; enquanto 41 (37\%) concordaram com a afirmativa: "Neste setor é difícil discutir sobre erros".

No domínio satisfação no trabalho, o item que obteve maior escore foi: "Eu gosto do meu trabalho", com 100 (91\%) concordâncias. Concordaram com o item "Este é um bom lugar para trabalhar" 87 (79\%) entrevistados (Quadro 1).

No que se refere ao domínio percepção do estresse, 92 (84\%) profissionais concordaram com a seguinte afirmativa: "Eu sou menos eficiente no trabalho quando estou cansado(a)". Concordaram com o item "Quando minha carga horária de trabalho é excessiva, meu desempenho é prejudicado" 82 (75\%) indivíduos; com "Eu tenho maior probabilidade de cometer erros em situações tensas e hostis", 79 (72\%) deles; e com "O cansaço prejudica meu desempenho em situações de emergência” 68 (72,0\%) entrevistados (Quadro 2).
Em relação ao domínio percepção da gerência da unidade/hospital, 27 (25\%) discordaram e 27 (25\%) foram neutros no tocante ao item "A administração da unidade apoia meus esforços diários". Apenas 32 (29\%) concordaram com a seguinte afirmativa: "Profissionais problemáticos da equipe são tratados de maneira construtiva pela administração da unidade"; 42 (38\%) concordaram com o item: "Recebo informações adequadas e oportunas da administração da unidade sobre eventos que podem afetar meu trabalho"; e 67 (61\%) concordaram com o item: "A administração da unidade está fazendo um bom trabalho" (Quadro 2).

No que tange ao domínio condição de trabalho, 75 (68\%) indivíduos concordaram com a seguinte afirmativa: "Neste setor, o número e a qualificação dos profissionais são suficientes para lidar com o número de pacientes". De acordo com a afirmativa "Toda a informação necessária para as decisões diagnósticas e terapêuticas está disponível rotineiramente para mim”, 39 (35\%) entrevistados discordaram (Quadro 2).

Os três domínios com maiores médias de escores foram: percepção do estresse, satisfação no trabalho e clima de trabalho em equipe, respectivamente. Somente os domínios percepção do estresse e satisfação no trabalho obtiveram médias com escores superiores a 75. O domínio menos estimado foi percepção da gerência do hospital ${ }^{10}$.

Quadro 1. Respostas para clima de trabalho em equipe, clima de segurança e satisfação no trabalho no centro cirúrgico.

\begin{tabular}{|l|c|c|c|c|}
\hline Domínio & $\begin{array}{c}\text { Discordo* } \\
\text { N (\%) }\end{array}$ & $\begin{array}{c}\text { Neutro } \\
\text { N (\%) }\end{array}$ & $\begin{array}{c}\text { Concordo* } \\
\text { N (\%) }\end{array}$ & $\begin{array}{c}\text { NA } \\
\text { N (\%) }\end{array}$ \\
\hline $\begin{array}{l}\text { Clima de trabalho em equipe } \\
\begin{array}{l}\text { Neste setor é difícil falar abertamente se eu percebo } \\
\text { um problema com o cuidado ao paciente }\end{array}\end{array}$ & $73(66)$ & $5(5)$ & $27(25)$ & $5(5)$ \\
$\begin{array}{l}\text { Eu tenho o apoio de que necessito dos outros } \\
\text { membros da equipe para cuidar dos pacientes } \\
\text { Clima de segurança }\end{array}$ & $13(12)$ & $0(0)$ & $90(82)$ & $7(6)$ \\
\hline $\begin{array}{l}\text { Erros são tratados de maneira apropriada neste setor } \\
\text { Neste setor é difícil discutir sobre erros }\end{array}$ & $23(21)$ & $18(16)$ & $64(58)$ & $5(5)$ \\
\hline $\begin{array}{l}\text { Satisfação no trabalho } \\
\text { Eu gosto do meu trabalho }\end{array}$ & $51(46)$ & $13(12)$ & $41(37)$ & $5(5)$ \\
\hline Este é um bom lugar para trabalhar & $2(2)$ & $2(2)$ & $100(91)$ & $6(5)$ \\
\hline
\end{tabular}

*Parcial ou totalmente; NA: não se aplica. 
Quadro 2. Respostas para percepção do estresse, percepção da gerência e condições de trabalho no centro cirúrgico.

\begin{tabular}{|c|c|c|c|c|}
\hline Domínio & $\begin{array}{l}\text { Discordo* } \\
\text { N (\%) }\end{array}$ & $\begin{array}{l}\text { Neutro } \\
\text { N (\%) }\end{array}$ & $\begin{array}{l}\text { Concordo* } \\
\text { N (\%) }\end{array}$ & $\begin{array}{l}\text { NA } \\
\text { N }(\%)\end{array}$ \\
\hline \multicolumn{5}{|l|}{ Percepção do estresse } \\
\hline $\begin{array}{l}\text { Quando minha carga horária de trabalho é excessiva, meu } \\
\text { desempenho é prejudicado. }\end{array}$ & $14(13)$ & $7(6)$ & $82(75)$ & $7(6)$ \\
\hline Eu sou menos eficiente no trabalho quando estou cansado(a). & $10(9)$ & 3 (3) & $92(84)$ & $5(5)$ \\
\hline $\begin{array}{l}\text { Eu tenho maior probabilidade de cometer erros em situações tensas } \\
\text { e hostis. }\end{array}$ & $18(16)$ & $8(7)$ & $79(72)$ & $5(5)$ \\
\hline O cansaço prejudica meu desempenho em situações de emergência. & $26(24)$ & $8(7)$ & $68(62)$ & $8(7)$ \\
\hline \multicolumn{5}{|l|}{ Percepção da gerência da unidade/hospital } \\
\hline A administração da unidade apoia meus esforços diários. & $27(25)$ & $27(25)$ & $47(43)$ & $9(8)$ \\
\hline A administração da unidade está fazendo um bom trabalho. & $16(15)$ & $18(16)$ & $67(61)$ & $9(8)$ \\
\hline $\begin{array}{l}\text { Profissionais problemáticos da equipe são tratados de maneira } \\
\text { construtiva pela administração da unidade. }\end{array}$ & $32(29)$ & $33(30)$ & $32(29)$ & $13(12)$ \\
\hline $\begin{array}{l}\text { Recebo informações adequadas e oportunas da administração da } \\
\text { unidade sobre eventos que podem afetar meu trabalho. }\end{array}$ & $36(33)$ & $20(18)$ & $42(38)$ & $12(11)$ \\
\hline \multicolumn{5}{|l|}{ Condições de trabalho } \\
\hline $\begin{array}{l}\text { Neste setor, o número e a qualificação dos profissionais são } \\
\text { suficientes para lidar com o número de pacientes. }\end{array}$ & $20(18)$ & 5 (5) & $75(68)$ & $10(9)$ \\
\hline $\begin{array}{l}\text { Toda a informação necessária para as decisões diagnósticas e } \\
\text { terapêuticas está disponível rotineiramente para mim. }\end{array}$ & $39(35)$ & $9(8)$ & $51(46)$ & $11(10)$ \\
\hline
\end{tabular}

*Parcial ou totalmente; NA: não se aplica.

\section{DISCUSSÃO}

Os resultados do estudo evidenciaram que aspectos relevantes da cultura de segurança do paciente no CC precisam ser aprimorados, uma vez que a avaliação da média dos escores confirma que apenas dois de seis domínios obtiveram valores superiores a 75 , sendo este considerado como mínimo positivo para a cultura de segurança.

Odomínio percepção do estresse, queéo reconhecimento pelos profissionais de elementos estressores na execução do seu trabalho, alcançou o maior escore no estudo e requer atenção. Destaca-se que os trabalhadores que participaram da pesquisa tiveram alta percepção das ocasiões estressantes no ambiente de trabalho, no entanto esse resultado é consistente com o de um estudo conduzido com profissionais no Distrito Federal para avaliar a cultura de segurança em um hospital público e que também demonstrou, por meio de escore positivo, que os colaboradores notam quando os fatores estressores interferem na execução do trabalho ${ }^{6}$.

A alta percepção dos fatores estressores no ambiente de trabalho também foi identificada em estudos internacionais realizados na Noruega e na Hungria que mostraram resultados semelhantes aos de estudos brasileiros, destacando que os profissionais apresentaram boa percepção dos fatores estressores no ambiente de trabalho ${ }^{6}$.
Como o estresse é reconhecidamente uma influência negativa em qualquer atividade humana, é crucial que o profissional se conscientize de que, para prestar cuidados ao paciente, primeiramente precisa cuidar de si, evitar sentimentos de estresse e angústia que o predispõem ao erro ${ }^{11}$.

Os profissionais têm ciência de que a carga horária excessiva e o cansaço prejudicam o desempenho e aumentam a probabilidade de ocorrência de erros em situações tensas e hostis, comprometendo, assim, a segurança do paciente. Com isso, pode-se afirmar que a maioria dos profissionais reconhece os fatores estressores como fadiga, insuficiência de recursos humanos, fator emocional e barreira de comunicação como algo a ser revertido positivamente ${ }^{10}$.

Satisfação no trabalho foi o segundo domínio com maior média de escores. Estudo feito com profissionais de enfermagem do Rio Grande do Sul se assemelha com o resultado da afirmação "Gosto do meu trabalho", revelando que $91 \%$ dos profissionais afirmaram que gostavam do trabalho que realizavam e $82 \%$ se orgulhavam de trabalhar na área ${ }^{11}$. A satisfação do profissional de saúde é considerada um fator positivo, já que implica diretamente a qualidade da assistência prestada ${ }^{12}$.

Profissionais descontentes apresentam taxas de rotatividade altas, associadas à ocorrência de eventos adversos, 
como erros na administração de medicamentos e incidência de quedas. Nesse sentido, a satisfação no trabalho correlaciona-se com o aumento na produtividade e na segurança do paciente, pois profissionais satisfeitos podem deixar o local de trabalho mais seguro ${ }^{13}$.

O trabalho em equipe é a junção de relação harmônica, interação e colaboração entre elementos no mesmo ambiente físico. O estudo demonstra o domínio clima de trabalho em equipe como satisfatório, um aspecto positivo na instituição estudada.

Os resultados obtidos no domínio clima de trabalho em equipe se assemelham aos de um estudo realizado no Ceará que indica que em um ambiente onde os participantes podem dar sugestões, discutir abertamente sobre algum problema, em que um auxilia o outro e médicos juntamente com enfermeiros trabalham de forma coordenada é favorecida a garantia da qualidade da assistência. Um ambiente de trabalho favorável depende de como os membros dessa equipe se relacionam. A harmonia e o respeito às diferenças são os principais fatores que levam ao desenvolvimento de um ambiente agradável, seguro e focado, acima de tudo, na segurança do paciente ${ }^{7}$.

Contudo, no mesmo domínio, os participantes apontaram que as discordâncias não são resolvidas de modo apropriado e que é difícil falar abertamente sobre um problema quanto ao cuidado do paciente, condição considerada negativa para a segurança, uma vez que a resolutividade de problemas fica prejudicada, facilitando a ocorrência de eventos adversos aos pacientes ${ }^{7}$.

O domínio clima de segurança apresentou média de escore inferior a 75, o que sugere que o entrosamento coletivo e o respeito entre os profissionais precisam ser fortalecidos, e $72 \%$ dos profissionais compartilharam a ideia de que se sentiriam seguros se fossem tratados nesse CC como pacientes, corroborando com os resultados de um estudo feito no sul do Brasil. Porém, ao mesmo tempo, relatam que é difícil discutir sobre erros e que esses erros não são tratados de maneira apropriada no setor ${ }^{10}$.

Cabe salientar que os escores significativamente baixos tanto da percepção da gerência da unidade quanto da percepção da gerência do hospital identificam a fragilidade da gestão hospitalar.

Os participantes não se sentem apoiados pela gestão hospitalar nem recebem informações adequadas que podem afetar o trabalho, configurando uma fragilidade na comunicação entre os gestores e os profissionais assistenciais. Estudo feito no sul do Brasil apresentou resultado semelhante ${ }^{13}$. Esse quesito requer muita atenção, pois reflete diretamente na qualidade do cuidado prestado. Esses resultados apontam para a necessidade de que as gerências têm de se debruçar sobre essas questões, pois não se construirá uma cultura de segurança se, essencialmente, as gerências da unidade e do hospital não estiverem envolvidas nem sensibilizadas ${ }^{12}$.

O domínio condições de trabalho obteve escore de 65,9, constatando a necessidade de realizar educação continuada e aperfeiçoamento das dificuldades para os profissionais ${ }^{14}$. Esse défice na educação continuada em saúde colabora para o baixo escore observado na percepção da gerência. Assim, os gestores devem promover e apoiar a implementação de práticas seguras baseadas em evidências científicas e protocolos de atendimento para promover uma assistência multiprofissional segura, diminuindo a chance de ocorrência de eventos adversos evitáveis.

\section{CONCLUSÃO}

O nível de cultura de segurança encontrado na instituição sede da pesquisa está abaixo do preconizado pela literatura. Os profissionais envolvidos identificaram que as ações gerenciais são consideradas o principal fator contribuinte para a fragilidade dessa cultura, entretanto demonstraram satisfação com o trabalho.

A análise dos dados proporcionou a realização de um diagnóstico situacional, servindo de alerta para os domínios que precisam ser trabalhados, como também para o desenvolvimento e o aprimoramento de estratégias que fortaleçam a cultura de segurança no CC. Sugerem-se intervenções consistentes nos domínios identificados, a fim de promover um ambiente seguro para os profissionais e para os pacientes.

Esses resultados podem ser úteis para os gestores dessa unidade hospitalar, pois, ao identificar o nível de cultura de segurança, ações podem ser planejadas para a promoção da segurança do paciente, como processos de educação continuada e introdução de novas ferramentas, como o checklist de segurança cirúrgica, proposto pela OMS.

Considerando que o estudo se concentrou no CC, recomenda-se que esta pesquisa seja replicada em outras unidades hospitalares, a fim de identificar as fragilidades e potencialidades de cada área de trabalho, visando a um planejamento de mudança baseado na avaliação dos profissionais que atuam diariamente no cuidado em saúde. 


\section{REFERÊNCIAS}

1. Barbosa M, Floriano D, Oliveira K, Nascimento K, Ferreira L. Clima de segurança do paciente em um hospital privado. Texto Contexto Enferm. 2016;25(3):e1460015. https://doi. org/10.1590/0104-07072016001460015

2. Brasil. Ministério da Saúde. Fundação Oswaldo Cruz. Agência Nacional de Vigilância Sanitária. Portaria n 529, de $1^{\circ}$ de abril de 2013 (DOU de 02/04/2013). Institui o Programa Nacional de Segurança do Paciente (PNSP) [Internet]. 2013 [acessado em 10 ago. 2018]. Disponível em: http://www.saude.mt.gov.br/upload/controle-infeccoes/pasta2/ portaria-msgm-n-529-de-01-04-2013.pdf

3. Cauduro F, Sarquis L, Sarquis L, Cruz E. Cultura de segurança entre profissionais de centro cirúrgico. Cogitare Enferm. 2015;20(1). http:// dx.doi.org/10.5380/ce.v20i1.36645

4. Brasil. Ministério da Saúde. Agência Nacional de Vigilância Sanitária (ANVISA). Resolução da Diretoria Colegiada (RDC) n 36, de 35 de julho de 2013. Institui ações para a segurança do paciente em serviços de saúde e dá outras providências. Diário Oficial da União. 2013.

5. Bohomol E, Tartali J. Eventos adversos em pacientes cirúrgicos: conhecimento dos profissionais de enfermagem. Acta Paul Enferm. 2013;26(4):376-81. http://dx.doi.org/10.1590/S0103-21002013000400012

6. Carvalho P, Göttems L, Pires M, Oliveira M. Cultura de segurança no centro cirúrgico de um hospital público, na percepção dos profissionais de saúde. Revista Latino-Am Enferm. 2015;23(6):1041-8. http:// dx.doi.org/10.1590/0104-1169.0669.2647

7. Carvalho R, Arruda L, Nascimento N, Sampaio R, Cavalcante M, Costa A. Avaliação da cultura de segurança em hospitais públicos no Brasil. Rev Latino-Am Enferm. 2017;25:e2849. http://dx.doi. org/10.1590/1518-8345.1600.2849
8. Carvalho R, Cassiani S. Questionário Atitudes de Segurança: adaptação transcultural do Safety Attitudes Questionnaire - Short Form 2006 para o Brasil. Rev Latino-Am Enferm 2012;20(3):575-82. http:// dx.doi.org/10.1590/S0104-11692012000300020

9. Sexton J, Helmreich R, Neilands T, Rowan K, Vella K, Boyden J, et al. The Safety Attitudes Questionnaire: psychometric properties, benchmarking data, and emerging research. BMC Health Serv Res [Internet]. 2006 [acessado em fev. 2018];6. Disponível em: http:// dx.doi.org/10.1186/1472-6963-6-44

10. Correggio T, Amante L, Barbosa S. Avaliação da cultura de segurança do paciente em centro cirúrgico. Rev SOBECC. 2014;19(2):67-73. http://dx.doi.org/10.4322/sobecc.2014.012

11. Toso G, Golle L, Magnago T, Herr G, Loro M, Aozane F, et al. Cultura de segurança do paciente em instituições hospitalares na perspectiva da enfermagem. Rev Gaúcha Enferm. 2016;37(4). http://dx.doi. org/10.1590/1983-1447.2016.04.58662

12. Rigobello M, Carvalho R, Cassiani S, Galon T, Capucho H, Deus N. Clima de segurança do paciente: percepção dos profissionais de enfermagem. Acta Paul Enferm. 2012;25(5):728-35. http://dx.doi. org/10.1590/S0103-21002012000500013

13. Fidelis R. Cultura de segurança: perspectiva da equipe de enfermagem em unidade de emergência do adulto [dissertação]. Florianópolis: Universidade Federal de Santa Catarina; 2011.

14. Marinho MM, Radünz V, Barbosa SFF. Avaliação da cultura de segurança pelas equipes de enfermagem de unidades cirúrgicas. Texto Contexto Enferm. 2014;23(3):581-90. http://dx.doi. org/10.1590/0104-07072014002640012 